\title{
Computing parametric rational generating functions with a primal Barvinok algorithm
}

\author{
Matthias Köppe* \\ Otto-von-Guericke-Universität Magdeburg, \\ Department of Mathematics, \\ Institute for Mathematical Optimization (IMO), \\ Universitätsplatz 2, \\ 39106 Magdeburg, Germany \\ mkoeppe@imo.math. uni-magdeburg. de \\ Sven Verdoolaege \\ Leiden Institute of Advanced Computer Science (LIACS), \\ Universiteit Leiden, \\ Niels Bohrweg 1, \\ 2333 CA Leiden, The Netherlands \\ sverdool@liacs.nl
}

Submitted: Aug 27, 2007; Accepted: Oct 5, 2007; Published: Jan 21, 2008

Mathematics Subject Classifications: 05A15; 52C07; 68W30

\begin{abstract}
Computations with Barvinok's short rational generating functions are traditionally being performed in the dual space, to avoid the combinatorial complexity of inclusion-exclusion formulas for the intersecting proper faces of cones. We prove that, on the level of indicator functions of polyhedra, there is no need for using inclusion-exclusion formulas to account for boundary effects: All linear identities in the space of indicator functions can be purely expressed using partially open variants of the full-dimensional polyhedra in the identity. This gives rise to a practically efficient, parametric Barvinok algorithm in the primal space.
\end{abstract}

\section{Introduction}

We consider a family of polytopes $P_{\mathbf{q}}=\left\{\mathbf{x} \in \mathbf{R}^{d}: A \mathbf{x} \leq \mathbf{q}\right\}$ parameterized by a righthand side vector $\mathbf{q} \in Q \subseteq \mathbf{R}^{m}$, where the set of right-hand sides is restricted to some

${ }^{*}$ The first author was supported by a 2006/2007 Feodor Lynen Research Fellowship from the Alexander von Humboldt Foundation. He also acknowledges the hospitality of Jesús De Loera and the Department of Mathematics of the University of California, Davis, where a part of this work was completed. 
polyhedron $Q$. For this family of polytopes, we define the parametric counting function $c: Q \rightarrow \mathbf{N}$ by

$$
c(\mathbf{q})=\#\left(P_{\mathbf{q}} \cap \mathbf{Z}^{d}\right) .
$$

Note that this includes vector partition functions $c(\boldsymbol{\lambda})=\#\left\{\mathbf{x} \in \mathbf{N}^{d}: A^{\prime} \mathbf{x}=\boldsymbol{\lambda}\right\}$ as a special case. It is well-known that the counting function (1) is a piecewise quasipolynomial function, i.e., a function that, within each of a finite set of polyhedra $Q_{i}$ that form a subdivision of $Q$ and for each residue class modulo a lattice in $Q_{i}$, behaves as a polynomial. We are interested in computing an efficient algorithmic representation of the function that allows to efficiently evaluate $c(\mathbf{q})$ for any given $\mathbf{q}$. This paper builds on various techniques described in the literature, which we review in the following.

\subsection{Barvinok's short rational generating functions}

The foundation of our method is an algorithmically efficient calculus of rational generating functions of the integer points in polyhedra developed by Barvinok [3]; see also [5]. Let $P=P_{\mathbf{q}} \subseteq \mathbf{R}^{d}$ be a rational polyhedron. By elimination of variables we may assume that $P$ is full-dimensional. The generating function of $P \cap \mathbf{Z}^{d}$ is defined as the formal Laurent series

$$
\tilde{g}_{P}(\mathbf{z})=\sum_{\boldsymbol{\alpha} \in P \cap \mathbf{Z}^{d}} \mathbf{z}^{\boldsymbol{\alpha}} \in \mathbf{Z}\left[\left[z_{1}, \ldots, z_{d}, z_{1}^{-1}, \ldots, z_{d}^{-1}\right]\right]
$$

using the multi-exponent notation $\mathbf{z}^{\boldsymbol{\alpha}}=\prod_{i=1}^{d} z_{i}^{\alpha_{i}}$. If $P$ is bounded, $\tilde{g}_{P}$ is a Laurent polynomial, which we consider as a rational function $g_{P}$. If $P$ is not bounded but is pointed (i.e., $P$ does not contain a straight line), there is a non-empty open subset $U \subseteq \mathbf{C}^{d}$ such that the series converges absolutely and uniformly on every compact subset of $U$ to a rational function $g_{P}$. If $P$ contains a straight line, the series does not converge, and we set $g_{P}=0$; this turns out to be the right choice to make the mapping $P \mapsto g_{P}$ a (rational-function-valued) valuation, i.e., a finitely additive measure [8]. The rational function $g_{P} \in \mathbf{Q}\left(z_{1}, \ldots, z_{d}\right)$ defined in this way is called the rational generating function of $P \cap \mathbf{Z}^{d}$.

By Brion's Theorem [8], the rational generating function of a polyhedron $P$ is the sum of the rational generating functions of its vertex cones, i.e., for each vertex $\mathbf{v}$ of $P$, the affine polyhedral cone $\left\{\mathbf{v}+\lambda \mathbf{y} \in \mathbf{R}^{d}: \lambda \in \mathbf{R}, \lambda \geq 0, \mathbf{v}+\mathbf{y} \in P\right\}$. Thus the computation of a rational generating function can be reduced to the case of affine polyhedral cones. Moreover, as mentioned above, the mapping $P \mapsto g_{P}$ is a valuation: Let $[P]$ denote the indicator function of $P$, i.e., the function

$$
[P]: \mathbf{R}^{d} \rightarrow \mathbf{R}, \quad[P](\mathbf{x})= \begin{cases}1 & \text { if } \mathbf{x} \in P \\ 0 & \text { otherwise }\end{cases}
$$

The valuation property is that any (finite) linear identity $\sum_{i \in I} \varepsilon_{i}\left[P_{i}\right]=0$ with $\varepsilon_{i} \in \mathbf{Q}$ carries over to a linear identity $\sum_{i \in I} \varepsilon_{i} g_{P_{i}}(\mathbf{z})=0$. Hence, it is possible to use the inclusionexclusion principle to break a polyhedral cone into pieces and to add and subtract the resulting generating functions. Indeed, by triangulating the vertex cones, one can reduce 
the problem to the case of simplicial cones, i.e., cones $C \subseteq \mathbf{R}^{d}$ generated by $d$ linearly independent ray vectors $\mathbf{b}_{1}, \ldots, \mathbf{b}_{d} \in \mathbf{Z}^{d}$.

The index of a (full-dimensional) simplicial cone is defined as the index of the point lattice generated by $\mathbf{b}_{1}, \ldots, \mathbf{b}_{d}$ in the standard lattice $\mathbf{Z}^{d}$; we have ind $C=\left|\operatorname{det}\left(\mathbf{b}_{1}, \ldots, \mathbf{b}_{d}\right)\right|$. Using Barvinok's signed decomposition technique, it is possible to write a cone as

$$
[C]=\sum_{i \in I_{1}} \varepsilon_{i}\left[C_{i}\right]+\sum_{i \in I_{2}} \varepsilon_{i}\left[C_{i}\right] \quad \text { with } \varepsilon_{i} \in\{ \pm 1\}
$$

with at most $d$ full-dimensional simplicial cones $C_{i}$ of lower index in the sum over $i \in I_{1}$ and $\mathrm{O}\left(2^{d}\right)$ lower-dimensional simplicial cones $C_{i}$ in the sum over $i \in I_{2}$. The lower-dimensional cones arise due to the inclusion-exclusion principle applied to the intersecting faces of the full-dimensional cones. The signed decomposition is then recursively applied to the cones $C_{i}$, until one obtains unimodular (index 1) cones, for which the rational generating function can be written down trivially. Since the indices of the full-dimensional cones descend quickly enough at each level of the decomposition, one can prove the depth of the decomposition tree is doubly logarithmic in the index of the input cone. This gives rise to a polynomiality result in fixed dimension:

Theorem 1 (Barvinok [3]). Let the dimension d be fixed. There exists a polynomialtime algorithm for computing the rational generating function of a polyhedron $P \subseteq \mathbf{R}^{d}$ given by rational inequalities.

Despite the polynomiality result, the algorithm was widely considered to be practically inefficient because too many, $\mathrm{O}\left(2^{d}\right)$, lower-dimensional cones had to be created at every level of the decomposition. Later the algorithm was improved by making use of Brion's "polarization trick", see [8] and [5, Remark 4.3]: The computations with rational generating functions are invariant with respect to the contribution of non-pointed cones (cones containing a non-trivial linear subspace). The reason is that the rational generating function of every non-pointed cone is zero. By operating in the dual space, i.e., by computing with the polars of all cones, lower-dimensional cones can be safely discarded, because this is equivalent to discarding non-pointed cones in the primal space. Thus at each level of the decomposition, only at most $d$ cones are created. This dual variant of Barvinok's algorithm has efficient implementations in LattE [10, 11, 12] and the library barvinok [21].

\subsection{Parametric polytopes and generating functions}

The vertices of a parametric polytope $P_{\mathbf{q}}=\left\{\mathbf{x} \in \mathbf{R}^{d}: A \mathbf{x} \leq \mathbf{q}\right\}$, with $\mathbf{q} \in Q \subseteq \mathbf{R}^{m}$ are affine functions of the parameters $\mathbf{q}$ and can be computed as follows. A set $B$ of $d$ linearly independent rows of the inequality system $A \mathbf{x} \leq \mathbf{q}$ is called a simplex basis. The associated basic solution $\mathbf{x}(B)$ is the unique solution of the equation $A_{B} \mathbf{x}=\mathbf{q}_{B}$. Note that different simplex bases may give rise to the same basic solution. A simplex basis (and the corresponding basic solution) is called (primal) feasible if $A \mathbf{x}(B) \leq \mathbf{q}$ holds for 
some $\mathbf{q} \in Q$. The vertices of $P_{\mathbf{q}}$ correspond to the feasible basic solutions and they are said to be active on the subset of $Q$ for which the basic solutions are feasible.

A chamber of the parameterized inequality system $A \mathbf{x} \leq \mathbf{q}$ is an inclusion-maximal set of right-hand side vectors $\mathbf{q}$ that have the same set of primal feasible simplex bases. The chamber complex of $P_{\mathbf{q}}$ is the common refinement of the projections into $Q$ of the $n$-faces of the polyhedron $\hat{P}=\left\{(\mathbf{x}, \mathbf{q}) \in \mathbf{R}^{d} \times Q: A \mathbf{x} \leq \mathbf{q}\right\}$, where $n$ is the dimension of the projection of $\hat{P}$ onto $Q[17,22]$. Alternatively, the problem may be translated into a vector partition problem, for which the chambers can be computed either directly [2] or as the regular triangulations of its Gale transform [14, 19]. However, these alternative computations, discussed in more detail in [13,21], may lead to many chambers that do not meet $Q$ and that hence have to be discarded.

Within each (open) chamber of the chamber complex, the combinatorial type of $P_{\mathbf{q}}$ remains the same and Barvinok's algorithm can be applied to the vertices active on the chamber [5, Theorem 5.3]. As we will explain in more detail in Section 3.1, the result is a parametric rational generating function where the parameters only appear in the numerator. In practice, it is sufficient to apply Barvinok's algorithm in the closures of the chambers of maximal dimension [9, Section 4.2]. On intersections of these closures one obtains possibly different representations of the same parametric rational generating function.

Example 2. As a trivial example, consider the one-dimensional parametric polytope $P_{q}=\left\{x \in \mathbf{R}^{1}: x \geq 0,2 x \leq q+6, x \leq q\right\}$. Its vertices are $0, q / 2+3$ and $q$, active on $\{q \geq 0\},\{q \geq 6\}$ and $\{q \leq 6\}$, respectively. The full-dimensional (open) chambers are $\{0<q<6\}$ and $\{q>6\}$ and the resulting parametric counting function is

$$
c(q)= \begin{cases}q+1 & \text { if } 0 \leq q \leq 6 \\ \left\lfloor\frac{q}{2}\right\rfloor+4 & \text { if } 6 \leq q .\end{cases}
$$

As in the non-parametric case, $P_{\mathbf{q}}$ can be assumed to be full-dimensional for all parameter values in the chambers of maximal dimension. Note that a reduction to the full-dimensional case may involve a reduction of the parameters to the standard lattice $[18,24]$. This parametric version of the dual variant of Barvinok's algorithm has also been implemented in barvinok [21] and is explained in more detail in [22, 23, 24].

\subsection{Irrational decompositions and primal algorithms}

Recently, Beck and Sottile [6] introduced irrational triangulations of polyhedral cones as a technique for obtaining simplified proofs for theorems on generating functions. Let $\mathbf{v}+C \subseteq \mathbf{R}^{d}$ be a full-dimensional affine polyhedral cone; it can be triangulated into simplicial full-dimensional cones $\mathbf{v}+C_{i}$. Then there exists a vector $\tilde{\mathbf{v}} \in \mathbf{R}^{d}$ such that

$$
(\tilde{\mathbf{v}}+C) \cap \mathbf{Z}^{d}=(\mathbf{v}+C) \cap \mathbf{Z}^{d}
$$

and

$$
\partial\left(\tilde{\mathbf{v}}+C_{i}\right) \cap \mathbf{Z}^{d}=\emptyset,
$$


that is, the affine cones $\tilde{\mathbf{v}}+C_{i}$ do not have any integer points in common. Thus, without using the inclusion-exclusion principle, one obtains an identity on the level of generating functions,

$$
g_{\mathbf{v}+C}(\mathbf{z})=g_{\tilde{\mathbf{v}}+C}(\mathbf{z})=\sum_{i} g_{\tilde{\mathbf{v}}+C_{i}}(\mathbf{z}) .
$$

Köppe [15] considered both irrational triangulations and irrational signed decompositions. He constructed a uniform irrational shifting vector $\tilde{\mathbf{v}}$ which ensures that (3) holds for all cones $\tilde{\mathbf{v}}+C_{i}$ that are created during the course of the recursive Barvinok decomposition method. The implementation of this method in a version of LattE [16] was the first practically efficient variant of Barvinok's algorithm that works in the primal space.

The benefits of a decomposition in the primal space are twofold. First, it allows to effectively use the method of stopped decomposition [15], where the recursive decomposition of the cones is stopped before unimodular cones are obtained. For certain classes of polyhedra, this technique reduces the running time by several orders of magnitude.

Second, for some classes of polyhedra such as the cross-polytopes, it is prohibitively expensive to compute triangulations of the vertex cones in the dual space. An all-primal algorithm [15] that computes both triangulations and signed decompositions in the primal space is therefore able to handle problem instances that cannot be solved with a dual algorithm in reasonable time.

\subsection{The contribution of this paper}

The irrationalization technique of $[6,15]$ can be viewed as a method of translating an inexact identity (i.e., an identity modulo the contribution of lower-dimensional cones) of indicator functions of full-dimensional cones,

$$
\sum_{i \in I} \varepsilon_{i}\left[\mathbf{v}_{i}+C_{i}\right] \equiv 0 \quad(\bmod \text { lower-dimensional cones })
$$

to an exact identity of rational generating functions,

$$
\sum_{i \in I} \varepsilon_{i} g_{\tilde{\mathbf{v}}_{i}+C_{i}}(\mathbf{z})=0
$$

We remark that this identity is not valid on the level of indicator functions. In contrast, in Section 2.1 we provide a general constructive method of translating an inexact identity (5) of indicator functions of full-dimensional cones to an exact identity of indicator functions of full-dimensional partially open cones,

$$
\sum_{i \in I} \varepsilon_{i}\left[\mathbf{v}_{i}+\tilde{C}_{i}\right]=0
$$

without increasing the number of summands in the identity.

This general result gives rise to methods of exact polyhedral subdivision of polyhedral cones (Section 2.2) and exact signed decomposition of partially open simplicial cones (Section 2.3). 
Since the rational generating function of partially open simplicial cones of low index can be written down easily (Section 3.1), we obtain new primal variants of Barvinok's algorithm. The new variants have simpler implementations than the primal irrational variant [15, Algorithm 5.1] and the all-primal irrational variant [15, Algorithm 6.4] because computations with large rational numbers can be replaced by simple, combinatorial rules.

The new variants based on exact decomposition in the primal space are particularly useful for parametric problems. The reason is that the method of constructing the partially open polyhedral cones only depends on the facet normals and is independent from the location of the parametric vertex. In contrast, the irrationalization technique needs to shift the parametric vertex by a vector $\mathbf{s}$ which needs to depend on the parameters. This is of particular importance for the case of the irrational all-primal algorithm, where the irrational shifting vector $\mathbf{s}$ needs to be constructed by solving a parametric linear program.

Moreover, the technique of exact decomposition can also be applied to the parameter space $Q$, obtaining a partition into partially open chambers $\tilde{Q}_{i}$. This gives rise to useful new representations of the parametric generating function $g_{P_{\mathbf{q}}}(\mathbf{z})$ (Section 3.2) and the counting function $c(\mathbf{q})$ (Section 3.3). We also introduce algorithmic representations of $g_{P_{\mathbf{q}}}(\mathbf{z})$ and $c(\mathbf{q})$ that make use of partially open activity domains of the parametric vertices. Its benefit is that it is of polynomial size and has polynomial evaluation time even when the dimension $m$ of the parameter space varies.

Taking all together, we obtain the first practically efficient parametric Barvinok algorithm in the primal space.

\section{Exact triangulations and signed decompositions into partially open polyhedra}

\subsection{Identities in the algebra of indicator functions, or: Inclusion-exclusion is not hard for boundary effects}

We first show that identities of indicator functions of full-dimensional polyhedra modulo lower-dimensional polyhedra can be translated to exact identities of indicator functions of full-dimensional partially open polyhedra.

Theorem 3. Let

$$
\sum_{i \in I_{1}} \varepsilon_{i}\left[P_{i}\right]+\sum_{i \in I_{2}} \varepsilon_{i}\left[P_{i}\right]=0
$$

be a (finite) linear identity of indicator functions of closed polyhedra $P_{i} \subseteq \mathbf{R}^{d}$, where the polyhedra $P_{i}$ are full-dimensional for $i \in I_{1}$ and lower-dimensional for $i \in I_{2}$, and where $\varepsilon_{i} \in \mathbf{Q}$. Let each closed polyhedron be given as

$$
P_{i}=\left\{\mathbf{x}:\left\langle\mathbf{b}_{i, j}^{*}, \mathbf{x}\right\rangle \leq \beta_{i, j} \text { for } j \in J_{i}\right\} .
$$


Let $\mathbf{y} \in \mathbf{R}^{d}$ be a vector such that $\left\langle\mathbf{b}_{i, j}^{*}, \mathbf{y}\right\rangle \neq 0$ for all $i \in I_{1} \cup I_{2}, j \in J_{i}$. For $i \in I_{1}$, we define the partially open polyhedron

$$
\begin{array}{r}
\tilde{P}_{i}=\left\{\mathbf{x} \in \mathbf{R}^{d}:\left\langle\mathbf{b}_{i, j}^{*}, \mathbf{x}\right\rangle \leq \beta_{i, j} \text { for } j \in J_{i} \text { with }\left\langle\mathbf{b}_{i, j}^{*}, \mathbf{y}\right\rangle<0,\right. \\
\left.\left\langle\mathbf{b}_{i, j}^{*}, \mathbf{x}\right\rangle<\beta_{i, j} \text { for } j \in J_{i} \text { with }\left\langle\mathbf{b}_{i, j}^{*}, \mathbf{y}\right\rangle>0\right\} .
\end{array}
$$

Then

$$
\sum_{i \in I_{1}} \varepsilon_{i}\left[\tilde{P}_{i}\right]=0
$$

Proof. We will show that (11) holds for an arbitrary $\overline{\mathbf{x}} \in \mathbf{R}^{d}$. To this end, fix an arbitrary $\overline{\mathbf{x}} \in \mathbf{R}^{d}$. We define

$$
\mathbf{x}_{\lambda}=\overline{\mathbf{x}}+\lambda \mathbf{y} \quad \text { for } \lambda \in[0,+\infty)
$$

Consider the function

$$
f:[0,+\infty) \ni \lambda \mapsto\left(\sum_{i \in I_{1}} \varepsilon_{i}\left[\tilde{P}_{i}\right]\right)\left(\mathbf{x}_{\lambda}\right) .
$$

We need to show that $f(0)=0$. To this end, we first show that $f$ is constant in a neighborhood of 0 .

First, let $i \in I_{1}$ such that $\overline{\mathbf{x}} \in \tilde{P}_{i}$. For $j \in J_{i}$ with $\left\langle\mathbf{b}_{i, j}^{*}, \mathbf{y}\right\rangle<0$, we have $\left\langle\mathbf{b}_{i, j}^{*}, \overline{\mathbf{x}}\right\rangle \leq$ $\beta_{i, j}$, thus $\left\langle\mathbf{b}_{i, j}^{*}, \mathbf{x}_{\lambda}\right\rangle \leq \beta_{i, j}$. For $j \in J_{i}$ with $\left\langle\mathbf{b}_{i, j}^{*}, \mathbf{y}\right\rangle>0$, we have $\left\langle\mathbf{b}_{i, j}^{*}, \overline{\mathbf{x}}\right\rangle<\beta_{i, j}$, thus $\left\langle\mathbf{b}_{i, j}^{*}, \mathbf{x}_{\lambda}\right\rangle<\beta_{i, j}$ for $\lambda>0$ small enough. Hence, $\mathbf{x}_{\lambda} \in \tilde{P}_{i}$ for $\lambda>0$ small enough.

Second, let $i \in I_{1}$ such that $\overline{\mathbf{x}} \notin \tilde{P}_{i}$. Then either there exists a $j \in J_{i}$ with $\left\langle\mathbf{b}_{i, j}^{*}, \mathbf{y}\right\rangle<0$ and $\left\langle\mathbf{b}_{i, j}^{*}, \overline{\mathbf{x}}\right\rangle>\beta_{i, j}$. Then $\left\langle\mathbf{b}_{i, j}^{*}, \mathbf{x}_{\lambda}\right\rangle>\beta_{i, j}$ for $\lambda>0$ small enough. Otherwise, there exists a $j \in J_{i}$ with $\left\langle\mathbf{b}_{i, j}^{*}, \mathbf{y}\right\rangle>0$ and $\left\langle\mathbf{b}_{i, j}^{*}, \overline{\mathbf{x}}\right\rangle \geq \beta_{i, j}$. Then $\left\langle\mathbf{b}_{i, j}^{*}, \mathbf{x}_{\lambda}\right\rangle \geq \beta_{i, j}$. Hence, in either case, $\mathbf{x}_{\lambda} \notin \tilde{P}_{i}$ for $\lambda>0$ small enough.

Next we show that $f$ vanishes on some interval $\left(0, \lambda_{0}\right)$. We consider the function

$$
g:[0,+\infty) \ni \lambda \mapsto\left(\sum_{i \in I_{1}} \varepsilon_{i}\left[P_{i}\right]+\sum_{i \in I_{2}} \varepsilon_{i}\left[P_{i}\right]\right)\left(\mathbf{x}_{\lambda}\right),
$$

which is constantly zero by (8). Since $\left[P_{i}\right]\left(\mathbf{x}_{\lambda}\right)$ for $i \in I_{2}$ vanishes on all but finitely many $\lambda$, we have

$$
g(\lambda)=\left(\sum_{i \in I_{1}} \varepsilon_{i}\left[P_{i}\right]\right)\left(\mathbf{x}_{\lambda}\right)
$$

for $\lambda$ from some interval $\left(0, \lambda_{1}\right)$. Also, $\left[P_{i}\right]\left(\mathbf{x}_{\lambda}\right)=\left[\tilde{P}_{i}\right]\left(\mathbf{x}_{\lambda}\right)$ for some interval $\left(0, \lambda_{2}\right)$. Hence $f(\lambda)=g(\lambda)=0$ for some interval $\left(0, \lambda_{0}\right)$.

Hence, since $f$ is constant in a neighborhood of 0 , it is also zero at $\lambda=0$. Thus the identity (11) holds for $\overline{\mathbf{x}}$.

Remark 4. Theorem 3 can be easily generalized to a situation where the weights $\varepsilon_{i}$ are not constants but continuous real-valued functions. In the proof, rather than showing that $f$ is constant in a neighborhood of 0 , one shows that $f$ is continuous at 0 . 


\subsection{The exact polyhedral subdivision of a closed polyhedral cone}

For obtaining an exact polyhedral subdivision of a full-dimensional closed polyhedral cone $C=\operatorname{cone}\left\{\mathbf{b}_{1}, \ldots, \mathbf{b}_{n}\right\}$,

$$
[C]=\sum_{i \in I_{1}}\left[\tilde{C}_{i}\right]
$$

we first compute a standard polyhedral subdivision,

$$
[C] \equiv \sum_{i \in I_{1}}\left[C_{i}\right] \quad(\bmod \text { lower-dimensional cones })
$$

where the lower-dimensional cones are proper faces of the full-dimensional cones. Then we apply the above theorem using an arbitrary vector $\mathbf{y} \in \operatorname{int} C$ that avoids all facets of the cones $C_{i}$, for instance

$$
\mathbf{y}=\sum_{i=1}^{n}\left(1+\gamma^{i}\right) \mathbf{b}_{i}
$$

for a suitable $\gamma>0$.

\subsection{The exact signed decomposition of partially open simplicial cones}

Let $\tilde{C} \subseteq \mathbf{R}^{d}$ be a partially open simplicial full-dimensional cone with the double description

$$
\begin{aligned}
& \tilde{C}=\left\{\mathbf{x} \in \mathbf{R}^{d}:\left\langle\mathbf{b}_{j}^{*}, \mathbf{x}\right\rangle \leq 0 \text { for } j \in J_{\leq} \text {and }\left\langle\mathbf{b}_{j}^{*}, \mathbf{x}\right\rangle<0 \text { for } j \in J_{<}\right\} \\
& \tilde{C}=\left\{\sum_{j=1}^{d} \lambda_{j} \mathbf{b}_{j}: \lambda_{j} \geq 0 \text { for } j \in J_{\leq} \text {and } \lambda_{j}>0 \text { for } j \in J_{<}\right\}
\end{aligned}
$$

where $J_{<} \cup J_{\leq}=\{1, \ldots, d\}$, with the biorthogonality property for the outer normal vectors $\mathbf{b}_{j}^{*}$ and the ray vectors $\mathbf{b}_{i}$,

$$
\left\langle\mathbf{b}_{j}^{*}, \mathbf{b}_{i}\right\rangle=-\delta_{i, j}= \begin{cases}-1 & \text { if } i=j \\ 0 & \text { otherwise }\end{cases}
$$

In the following we introduce a generalization of Barvinok's signed decomposition [3] to partially open simplicial cones $C_{i}$, which will give an exact identity of partially open cones. To this end, we first compute the usual signed decomposition of the closed cone $C=\operatorname{cl} \tilde{C}$,

$$
[C] \equiv \sum_{i} \varepsilon_{i}\left[C_{i}\right] \quad(\bmod \text { lower-dimensional cones })
$$

using an extra ray w, which has the representation

$$
\mathbf{w}=\sum_{i=1}^{d} \alpha_{i} \mathbf{b}_{i} \quad \text { where } \alpha_{i}=-\left\langle\mathbf{b}_{i}^{*}, \mathbf{w}\right\rangle
$$


Each of the cones $C_{i}$ is spanned by $d$ vectors from the set $\left\{\mathbf{b}_{1}, \ldots, \mathbf{b}_{d}, \mathbf{w}\right\}$. The signs $\varepsilon_{i} \in\{ \pm 1\}$ are determined according to the location of $\mathbf{w}$, see [3].

An exact identity

$$
[\tilde{C}]=\sum_{i} \varepsilon_{i}\left[\tilde{C}_{i}\right] \quad \text { with } \varepsilon \in\{ \pm 1\}
$$

can now be obtained from (15) as follows. We define cones $\tilde{C}_{i}$ that are partially open counterparts of $C_{i}$. We only need to determine which of the defining inequalities of the cones $\tilde{C}_{i}$ should be strict. To this end, we first show how to construct a vector $\mathbf{y}$ that characterizes which defining inequalities of $\tilde{C}$ are strict by the means of (10).

Lemma 5. Let

$$
\sigma_{i}= \begin{cases}1 & \text { for } i \in J_{\leq}, \\ -1 & \text { for } i \in J_{<},\end{cases}
$$

and let $\mathbf{y} \in R=\operatorname{int}$ cone $\left\{\sigma_{1} \mathbf{b}_{i}, \ldots, \sigma_{d} \mathbf{b}_{d}\right\}$ be arbitrary. Then

$$
\begin{aligned}
& J_{\leq}=\left\{j \in\{1, \ldots, d\}:\left\langle\mathbf{b}_{j}^{*}, \mathbf{y}\right\rangle<0\right\}, \\
& J_{<}=\left\{j \in\{1, \ldots, d\}:\left\langle\mathbf{b}_{j}^{*}, \mathbf{y}\right\rangle>0\right\} .
\end{aligned}
$$

We remark that the construction of such a vector $\mathbf{y}$ is not possible for a partially open non-simplicial cone in general.

Proof of Lemma 5. Such a $\mathbf{y}$ has the representation

$$
\mathbf{y}=\sum_{i \in J_{\leq}} \lambda_{i} \mathbf{b}_{i}-\sum_{i \in J_{<}} \lambda_{i} \mathbf{b}_{i} \quad \text { with } \lambda_{i}>0 .
$$

Thus

$$
\left\langle\mathbf{b}_{j}^{*}, \mathbf{y}\right\rangle= \begin{cases}-\lambda_{j} & \text { for } j \in J_{\leq} \\ +\lambda_{j} & \text { for } j \in J_{<}\end{cases}
$$

which proves the claim.

Now let $\mathbf{y} \in R$ be an arbitrary vector that is not orthogonal to any of the facets of the cones $\tilde{C}_{i}$. Then such a vector $\mathbf{y}$ can determine which of the defining inequalities of the cones $\tilde{C}_{i}$ are strict.

In the following, we give a specific construction of such a vector $\mathbf{y}$. To this end, let $\mathbf{b}_{m}$ be the unique ray of $\tilde{C}$ that is not a ray of $\tilde{C}_{i}$. Then we denote by $\tilde{\mathbf{b}}_{0, m}^{*}$ the outer normal vector of the unique facet of $\tilde{C}_{i}$ not incident to $\mathbf{w}$. Now consider any facet $F$ of a cone $\tilde{C}_{i}$ that is incident to $\mathbf{w}$. Since $\tilde{C}_{i}$ is simplicial, there is exactly one ray of $\tilde{C}_{i}$, say $\mathbf{b}_{l}$, not incident to $F$. The outer normal vector of the facet is therefore characterized up to scale by the indices $l$ and $m$; thus we denote it by $\tilde{\mathbf{b}}_{l, m}^{*}$. See Figure 1 for an example of this naming convention. 
Let $\mathbf{b}_{0}=\mathbf{w}$. Then, for every outer normal vector $\tilde{\mathbf{b}}_{l, m}^{*}$ and every ray $\mathbf{b}_{i}, i=0, \ldots, d$, we have

$$
\beta_{i ; l, m}:=-\left\langle\tilde{\mathbf{b}}_{l, m}^{*}, \mathbf{b}_{i}\right\rangle \begin{cases}>0 & \text { for } i=l, \\ =0 & \text { for } i \neq l, m, \\ \in \mathbf{R} & \text { for } i=m .\end{cases}
$$

Now the outer normal vector has the representation

$$
\tilde{\mathbf{b}}_{l, m}^{*}=\sum_{i=1}^{d} \beta_{i ; l, m} \mathbf{b}_{i}^{*} .
$$

The conditions of (18) determine the outer normal vector $\tilde{\mathbf{b}}_{l, m}^{*}$ up to scale. For the normals $\tilde{\mathbf{b}}_{0, m}^{*}$, we can choose

$$
\tilde{\mathbf{b}}_{0, m}^{*}=\alpha_{m} \mathbf{b}_{m}^{*}
$$

For the other facets $\tilde{\mathbf{b}}_{l, m}^{*}$, we can choose

$$
\tilde{\mathbf{b}}_{l, m}^{*}=\left|\alpha_{m}\right| \mathbf{b}_{l}^{*}-\operatorname{sign} \alpha_{m} \cdot \alpha_{l} \mathbf{b}_{m}^{*}
$$

Now consider

$$
\mathbf{y}=\sum_{i=1}^{d} \sigma_{i}\left(\left|\alpha_{i}\right|+\gamma^{i}\right) \mathbf{b}_{i},
$$

which lies in the cone $R$ for every $\gamma>0$. We obtain

$$
\left\langle\tilde{\mathbf{b}}_{0, m}^{*}, \mathbf{y}\right\rangle=-\sigma_{m} \alpha_{m}\left(\left|\alpha_{m}\right|+\gamma^{m}\right)
$$

and

$$
\begin{aligned}
\left\langle\tilde{\mathbf{b}}_{l, m}^{*}, \mathbf{y}\right\rangle= & \left|\alpha_{m}\right|\left\langle\mathbf{b}_{l}^{*}, \mathbf{y}\right\rangle-\operatorname{sign} \alpha_{m} \cdot \alpha_{l}\left\langle\mathbf{b}_{m}^{*}, \mathbf{y}\right\rangle \\
= & -\left|\alpha_{m}\right| \sigma_{l}\left(\left|\alpha_{l}\right|+\gamma^{l}\right)+\operatorname{sign} \alpha_{m} \cdot \alpha_{l} \sigma_{m}\left(\left|\alpha_{m}\right|+\gamma^{m}\right) \\
= & \left(\operatorname{sign}\left(\alpha_{l} \alpha_{m}\right) \sigma_{m}-\sigma_{l}\right)\left|\alpha_{l}\right|\left|\alpha_{m}\right| \\
& \quad-\sigma_{l}\left|\alpha_{m}\right| \gamma^{l}+\operatorname{sign}\left(\alpha_{l} \alpha_{m}\right) \sigma_{m}\left|\alpha_{l}\right| \gamma^{m},
\end{aligned}
$$

for $l \neq 0$. The right-hand side of (23), as a polynomial in $\gamma$, only has finitely many roots. Thus there are only finitely many values of $\gamma$ for which a scalar product $\left\langle\tilde{\mathbf{b}}_{l, m}^{*}, \mathbf{y}\right\rangle$ can vanish for any of the finitely many facet normals $\tilde{\mathbf{b}}_{l, m}^{*}$. Let $\gamma>0$ be an arbitrary number for which none of the scalar products vanishes. Then the vector $\mathbf{y}$ defined by (21) determines which of the defining inequalities of the cones $\tilde{C}_{i}$ should be strict.

Remark 6. It is possible to construct an a-priori vector $\mathbf{y}$ that is suitable to determine which defining inequalities are strict for all the cones that arise in the hierarchy of triangulations and signed decompositions of a cone $C=\operatorname{cone}\left\{\mathbf{b}_{1}, \ldots, \mathbf{b}_{n}\right\}$ in Barvinok's algorithm. The construction uses the methods from [15]. Let $0<r \in \mathbf{Z}$ and $\hat{\mathbf{y}} \in \frac{1}{r} \mathbf{Z}^{d}$ and 
such that the open cube $\hat{\mathbf{y}}+B_{\infty}\left(\frac{1}{r}\right)$ is contained in $C$. (For instance, choose $\hat{\mathbf{y}}=\sum_{i=1}^{n} \mathbf{b}_{i}$ and choose $r$ large enough.) Let $D$ be an upper bound on the determinant of any simplicial cone that can arise in a triangulation of $C$, for instance

$$
D=\left(\max _{i=1}^{n}\left\|\mathbf{b}_{i}\right\|^{2}\right)^{n / 2}
$$

by Lemma 16 of [15]. Let $C=\max _{i=1}^{n}\left\|\mathbf{b}_{i}\right\|_{\infty}$. Using the data from Theorem 11 of [15],

$$
k=\left\lfloor 1+\frac{\log _{2} \log _{2} D}{\log _{2} \frac{d}{d-1}}\right\rfloor, \quad M=2(d-1) !\left(d^{k} C\right)^{d-1},
$$

we define

$$
\mathbf{s}=\frac{1}{r} \cdot\left(\frac{1}{(2 M)^{1}}, \frac{1}{(2 M)^{2}}, \ldots, \frac{1}{(2 M)^{d}}\right) .
$$

Finally let $\mathbf{y}=\hat{\mathbf{y}}+\mathbf{s}$. Then $\left\langle\mathbf{b}^{*}, \mathbf{y}\right\rangle \neq 0$ for any of the facet normals $\mathbf{b}^{*}$ that can arise in the hierarchy of triangulations and signed decompositions of the cone $C$.

Remark 7. For performing the exact signed decomposition in a software implementation, it is not actually necessary to construct the vector $\mathbf{y}$ and to evaluate scalar products. In the following, we show that we can devise simple, "combinatorial" rules to decide which defining inequalities should be strict. To this end, let $\gamma>0$ in (21) be small enough that none of the signs

$$
\sigma_{l, m}=-\operatorname{sign}\left\langle\tilde{\mathbf{b}}_{l, m}^{*}, \mathbf{y}\right\rangle
$$

given by (23) change if $\gamma$ is decreased even more. We can now determine $\sigma_{l, m}$ for all possible cases.

Case 0: $\alpha_{m}=0$. The cone would be lower-dimensional in this case, since $\mathbf{w}$ lies in the space spanned by the ray vectors except $\mathbf{b}_{m}$, and is hence discarded.

Case 1: $l=0$. From (22), we have

$$
\sigma_{0, m}=\operatorname{sign}\left(\alpha_{m}\right) \sigma_{m}
$$

Case 2: $l \neq 0, \alpha_{l}=0, \alpha_{m} \neq 0$. Here we have $\left\langle\tilde{\mathbf{b}}_{l, m}^{*}, \mathbf{y}\right\rangle=-\sigma_{l}\left|\alpha_{m}\right| \gamma^{l}$, thus

$$
\sigma_{l, m}=\sigma_{l}
$$

Case 3: $l \neq 0, \alpha_{l} \alpha_{m}>0$. In this case (23) simplifies to

$$
\left\langle\tilde{\mathbf{b}}_{l, m}^{*}, \mathbf{y}\right\rangle=\left(\sigma_{m}-\sigma_{l}\right)\left|\alpha_{l}\right|\left|\alpha_{m}\right|-\sigma_{l}\left|\alpha_{m}\right| \gamma^{l}+\sigma_{m}\left|\alpha_{l}\right| \gamma^{m}
$$

Case $3 a: \sigma_{l}=\sigma_{m}$. Here the first term of (25) cancels, so

$$
\sigma_{l, m}=-\operatorname{sign}\left\langle\tilde{\mathbf{b}}_{l, m}^{*}, \mathbf{y}\right\rangle= \begin{cases}1 & \text { if } l<m \\ -1 & \text { if } l>m\end{cases}
$$




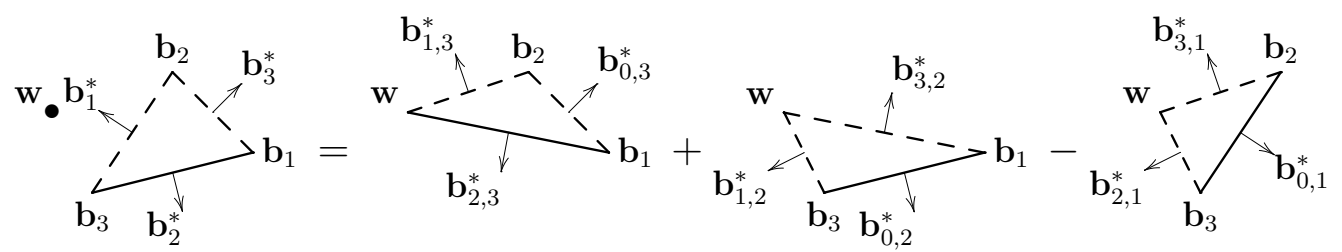

Figure 1: Signed decomposition of a partially open 3-dimensional simplicial cone. Each cone is represented by a vertex figure. Closed facets $(\sigma=1)$ are shown in solid lines, while open facets $(\sigma=-1)$ are shown in broken lines.

Case 3b: $\sigma_{l} \neq \sigma_{m}$. Here the first term of (25) dominates, so

$$
\sigma_{l, m}=-\operatorname{sign}\left\langle\tilde{\mathbf{b}}_{l, m}^{*}, \mathbf{y}\right\rangle=\sigma_{l} .
$$

Case $4: l \neq 0, \alpha_{l} \alpha_{m}<0$. In this case (23) simplifies to

$$
\left\langle\tilde{\mathbf{b}}_{l, m}^{*}, \mathbf{y}\right\rangle=-\left(\sigma_{m}+\sigma_{l}\right)\left|\alpha_{l}\right|\left|\alpha_{m}\right|-\sigma_{l}\left|\alpha_{m}\right| \gamma^{l}-\sigma_{m}\left|\alpha_{l}\right| \gamma^{m} .
$$

Case $4 a: \sigma_{l}=\sigma_{m}$. Here the first term of (26) dominates, so

$$
\sigma_{l, m}=\sigma_{l}=\sigma_{m} .
$$

Case $4 b: \sigma_{l} \neq \sigma_{m}$. Here the first term of (26) cancels, so

$$
\sigma_{l, m}=-\operatorname{sign}\left\langle\tilde{\mathbf{b}}_{l, m}^{*}, \mathbf{y}\right\rangle= \begin{cases}\sigma_{l} & \text { if } l<m, \\ \sigma_{m} & \text { if } l>m .\end{cases}
$$

Example 8. Consider (the vertex figure of) the three-dimensional cone on the left of Figure 1. The open and closed facets can be described as

$$
\sigma_{1}=-1 \quad \sigma_{2}=1 \quad \sigma_{3}=-1,
$$

while the extra ray $\mathbf{w}=\sum_{i=1}^{d} \alpha_{i} \mathbf{b}_{i}$ is such that

$$
\begin{array}{ccc}
\alpha_{1}<0 & \alpha_{2}>0 \quad \alpha_{3}>0 .
\end{array}
$$

For the facets of the cones in the decomposition we have

$$
\begin{array}{lll}
\sigma_{0,3} \stackrel{1}{=} \sigma_{3}=-1 & \sigma_{0,2} \stackrel{1}{=} \sigma_{2}=1 & \sigma_{0,1} \stackrel{1}{=}-\sigma_{1}=1 \\
\sigma_{1,3} \stackrel{4 a}{=} \sigma_{1}=-1 & \sigma_{1,2} \stackrel{4 b}{=} \sigma_{1}=-1 & \sigma_{2,1} \stackrel{4 b}{=} \sigma_{1}=-1 \\
\sigma_{2,3} \stackrel{3 b}{=} \sigma_{2}=1 & \sigma_{3,2} \stackrel{3 b}{=} \sigma_{3}=-1 & \sigma_{3,1} \stackrel{4 a}{=} \sigma_{1}=-1 .
\end{array}
$$

The result is shown on the right of Figure 1 .

Remark 9. Other constructions of $\mathbf{y}$ are possible, giving rise to different combinatorial rules. For instance, the implementation barvinok [21] uses a set of rules that correspond to a modification of (21), where for all $i \in J_{\leq}$the coefficient $\gamma^{i}$ is replaced by $\gamma^{i+d}$. 
Remark 10. After submission of this paper to the journal, we became aware that the above exact signed decomposition technique is related to a signed decomposition algorithm in the primal space based on Brion and Vergne [9]. The technique is based on an identity of indicator functions of simplicial cones modulo indicator functions of cones containing lines and was recently used by Berline and Vergne in their implementation of the computation of the $\mu$-functions in their local Euler-Maclaurin formulae [1, 7]. Using our technique, we can obtain a similar primal signed decomposition of closed cones as follows. Let us consider the exact signed decomposition $[\tilde{C}]=\sum_{i} \varepsilon_{i}\left[\tilde{C}_{i}\right]$ of a partially open simplicial cone $\tilde{C}$ into partially open simplicial cones $\tilde{C}_{i}$. On the level of rational generating functions (but not on the level of indicator functions), it is possible to replace each partially open cone in the identity by a closed cone as follows. Let $\tilde{C}$ be a partially open simplicial cone and let $\left\langle\mathbf{b}_{j}^{*}, \mathbf{x}\right\rangle<0$ be one of its strict inequalities. By replacing the strict inequality by the weak inequality $\left\langle\mathbf{b}_{j}^{*}, \mathbf{x}\right\rangle \geq 0$, we define a cone $\tilde{C}^{\prime}$. Since $\tilde{C} \cup \tilde{C}^{\prime}$ is a non-pointed cone, we have $g_{\tilde{C}^{\prime}}(\mathbf{z})=-g_{\tilde{C}}(\mathbf{z})$. By iterating this procedure, we obtain a closed cone $C^{\prime}$. Thus we can construct an identity of rational generating functions of closed simplicial cones, $g_{C^{\prime}}(\mathbf{z})=\sum_{i} \varepsilon_{i}^{\prime} g_{C_{i}^{\prime}}(\mathbf{z})$ with modified signs $\varepsilon^{\prime} \in\{ \pm 1\}$.

\section{Parametric Barvinok algorithm using exact decom- positions in the primal space}

In the previous section, we have shown how to both triangulate a closed polyhedral cone (Section 2.2) and apply Barvinok's decomposition (Section 2.3) in the primal space without introducing (indicator functions of) lower-dimensional polytopes. The result is a signed sum of partially open simplicial cones. The final remaining step in obtaining a generating function for a polytope is therefore the computation of the generating function of such a cone.

\subsection{The generating function of a partially open simplicial ratio- nal cone}

If $\mathbf{v}(\mathbf{q})+C$ is a closed simplicial affine cone where $C=\left\{\sum_{j=1}^{d} \lambda_{j} \mathbf{b}_{j}: \lambda_{j} \geq 0\right\}$ with $\mathbf{b}_{j} \in \mathbf{Z}^{d}$, then it is well known [20] that the generating function $g_{\mathbf{v}(\mathbf{q})+C}$ of $\mathbf{v}(\mathbf{q})+C$ is

$$
g_{\mathbf{v}(\mathbf{q})+C}(\mathbf{z})=\frac{\sum_{\boldsymbol{\alpha} \in \Pi \cap \mathbf{Z}^{d}} \mathbf{z}^{\boldsymbol{\alpha}}}{\prod_{j=1}^{d}\left(1-\mathbf{z}^{\mathbf{b}_{j}}\right)},
$$

with

$$
\Pi=\mathbf{v}(\mathbf{q})+\left\{\sum_{j=1}^{d} \lambda_{j} \mathbf{b}_{j}: 0 \leq \lambda_{j}<1\right\}
$$


the fundamental parallelepiped of $\mathbf{v}(\mathbf{q})+C$. For a partially open cone $\mathbf{v}(\mathbf{q})+\tilde{C}$ given by (13), the same formula holds with

$$
\Pi=\mathbf{v}(\mathbf{q})+\left\{\sum_{j=1}^{d} \lambda_{j} \mathbf{b}_{j}: 0 \leq \lambda_{j}<1 \text { for } j \in J_{\leq} \text {and } 0<\lambda_{j} \leq 1 \text { for } j \in J_{<}\right\} .
$$

To enumerate all points in $\Pi \cap \mathbf{Z}^{d}$ and compute the numerator of (27), we follow the technique of [4, Lemma 5.1], which we adapt for the case of partially open cones.

Lemma 11. Let $B$ be the matrix with the $\mathbf{b}_{j}$ as columns and let $S$ be the Smith normal form of $B$, i.e., $B V=W S$, with $V$ and $W$ unimodular matrices and $S$ a diagonal matrix $S=\operatorname{diag} \mathbf{s}$. Then,

$$
\Pi \cap \mathbf{Z}^{d}=\left\{\boldsymbol{\alpha}(\mathbf{k}): k_{j} \in \mathbf{Z}, 0 \leq k_{j}<s_{j}\right\}
$$

with

$$
\begin{aligned}
\boldsymbol{\alpha}(\mathbf{k}) & =\mathbf{v}(\mathbf{q})+\sum_{j \in J_{\leq}}\left\{\left\langle\mathbf{b}_{j}^{*}, \mathbf{v}(\mathbf{q})-W \mathbf{k}\right\rangle\right\} \mathbf{b}_{j}+\sum_{j \in J_{<}}\left\{\left\{\left\langle\mathbf{b}_{j}^{*}, \mathbf{v}(\mathbf{q})-W \mathbf{k}\right\rangle\right\}\right\} \mathbf{b}_{j} \\
& =W \mathbf{k}-\sum_{j \in J_{\leq}}\left\lfloor\left\langle\mathbf{b}_{j}^{*}, \mathbf{v}(\mathbf{q})-W \mathbf{k}\right\rangle\right\rfloor \mathbf{b}_{j}-\sum_{j \in J_{<}}\left\lceil\left\langle\mathbf{b}_{j}^{*}, \mathbf{v}(\mathbf{q})-W \mathbf{k}\right\rangle-1\right\rceil \mathbf{b}_{j},
\end{aligned}
$$

with $\{\cdot\}$ the (lower) fractional part $\{x\}=x-\lfloor x\rfloor$ and $\{\{\cdot\}\}$ the (upper) fractional part $\{\{x\}\}=x-\lceil x-1\rceil=1-\{-x\}$.

Proof. It is clear that each $\boldsymbol{\alpha}(\mathbf{k}) \in \Pi \cap \mathbf{Z}^{d}$. To see that all integer points in $\Pi$ are exhausted, note that $\operatorname{det} B=\operatorname{det} S$ and that all $\boldsymbol{\alpha}(\mathbf{k})$ are distinct. The latter follows from the fact that $\boldsymbol{\alpha}(\mathbf{k})$ can be written as $\boldsymbol{\alpha}(\mathbf{k})=W \mathbf{k}+B \boldsymbol{\gamma}=W \mathbf{k}+W S V^{-1} \boldsymbol{\gamma}$ for some $\boldsymbol{\gamma} \in \mathbf{Z}^{d}$. If $\boldsymbol{\alpha}\left(\mathbf{k}_{1}\right)=\boldsymbol{\alpha}\left(\mathbf{k}_{2}\right)$, we must therefore have $\mathbf{k}_{1} \equiv \mathbf{k}_{2}(\bmod \mathbf{s})$, i.e., $\mathbf{k}_{1}=\mathbf{k}_{2}$.

\subsection{Representations of the generating function of a parametric polytope}

Let $Q_{1}, \ldots, Q_{k} \subseteq Q$ be the chambers of the parameterized inequality system $A \mathbf{x} \leq \mathbf{q}$ of maximal dimension. For all parameters $\mathbf{q}$ from any given chamber $Q_{i}$, the parametric polytope $P_{\mathbf{q}}=\left\{\mathbf{x} \in \mathbf{R}^{d}: A \mathbf{x} \leq \mathbf{q}\right\}$ has the same set of primal feasible simplex bases. Due to affine-linear dependencies in the set $Q$ of parameters, several primal feasible simplex bases can yield the same vertex of the polytope $P_{\mathbf{q}}$ on the whole chamber $Q_{i}$. By this mapping we obtain a set $V_{i}$ of parametric vertices $\mathbf{v}_{j}(\mathbf{q})$ for $j \in V_{i}$ and associated vertex cones $\mathbf{v}_{j}(\mathbf{q})+C_{j}$. Let us denote by $g_{\mathbf{v}_{j}(\mathbf{q})+C_{j}}(\mathbf{z})$ the parametric generating function of the vertex cone at $\mathbf{v}_{j}(\mathbf{q})$.

By Brion's Theorem, we obtain the expression

$$
g_{P_{\mathbf{q}}}(\mathbf{z})=\sum_{\mathbf{v}_{j} \in V_{i}} g_{\mathbf{v}_{j}(\mathbf{q})+C_{j}}(\mathbf{z})
$$


for the generating function of the parametric polytope $P_{\mathbf{q}}$, valid for all parameters $\mathbf{q} \in Q_{i}$. It turns out [9, Section 4.2] that the formula (28) is also valid on the closure $\operatorname{cl} Q_{i}$ of the chamber $Q_{i}$. In this way, we obtain the usual representation of the parametric generating function as a piecewise function defined on the whole parameter space $Q$ :

$$
g_{P_{\mathbf{q}}}(\mathbf{z})=\left\{\begin{array}{cl}
\sum_{j \in V_{1}} g_{\mathbf{v}_{j}(\mathbf{q})+C_{j}}(\mathbf{z}) & \text { if } \mathbf{q} \in \operatorname{cl} Q_{1} \\
\vdots & \\
\sum_{j \in V_{k}} g_{\mathbf{v}_{j}(\mathbf{q})+C_{j}}(\mathbf{z}) & \text { if } \mathbf{q} \in \operatorname{cl} Q_{k}
\end{array}\right.
$$

As explained in Section 1.2, this yields possibly different expressions for values of $\mathbf{q}$ on the intersecting boundaries of two or more chambers.

We are now interested in a different representation of the parametric generating function,

$$
g_{P_{\mathbf{q}}}(\mathbf{z})=\left\{\begin{array}{cl}
\sum_{j \in V_{1}} g_{\mathbf{v}_{j}(\mathbf{q})+C_{j}}(\mathbf{z}) & \text { if } \mathbf{q} \in \tilde{Q}_{1} \\
\vdots & \\
\sum_{j \in V_{k}} g_{\mathbf{v}_{j}(\mathbf{q})+C_{j}}(\mathbf{z}) & \text { if } \mathbf{q} \in \tilde{Q}_{k},
\end{array}\right.
$$

where the sets $\tilde{Q}_{i}$ form a partition of the parameter space,

$$
Q=\tilde{Q}_{1} \cup \cdots \cup \tilde{Q}_{k} \quad \text { with } \quad \tilde{Q}_{i} \cap \tilde{Q}_{i}^{\prime}=\emptyset \text { for } i \neq i^{\prime} .
$$

The benefit of representation (30) is that it can be rewritten in the form of a closed formula using indicator functions,

$$
g_{P_{\mathbf{q}}}(\mathbf{z})=\sum_{i=1}^{k}\left[\tilde{Q}_{i}\right](\mathbf{q}) \sum_{j \in V_{i}} g_{\mathbf{v}_{j}(\mathbf{q})+C_{j}}(\mathbf{z}) .
$$

Clearly, representations (30) and (32) can be obtained by taking the chambers of all dimensions, since they form a partition of $Q$. However, we can do better:

Lemma 12. We can construct representations (30) and (32), where $k$ is the number of chambers of $A \mathbf{x} \leq \mathbf{q}$ of maximal dimension. When the dimension $d$ of the polytopes and the dimension $m$ of the parameter space are fixed, the construction is possible in polynomial time.

Proof. Again, we can apply the technique of Theorem 3 to define partially open polyhe$\operatorname{dra} \tilde{Q}_{i}$ that satisfy (31), where y is now an arbitrary vector from the relative interior of one of the chambers of maximal dimension. The complexity in fixed dimensions $m$ and $d$ follows from the fact that there are only polynomially many full-dimensional chambers in this case.

Note that the generating function of a parametric vertex may appear multiple times in representation (32) since a vertex $\mathbf{v}_{j}(\mathbf{q})$ may be active on more than one chamber. The multiple occurrences can be removed by considering the activity regions

$$
A_{j}=\left\{\mathbf{q}: A \mathbf{v}_{j}(\mathbf{q}) \leq \mathbf{q}\right\}
$$


of individual vertices instead of the chambers. Then, by introducing their partially open counterparts $\tilde{A}_{j}$ constructed by Theorem 3 , we obtain another representation of the parametric generating function,

$$
g_{P_{\mathbf{q}}}(\mathbf{z})=\sum_{j \in V}\left[\tilde{A}_{j}\right](\mathbf{q}) g_{\mathbf{v}_{j}(\mathbf{q})+C_{j}}(\mathbf{z}) .
$$

where $V=V_{1} \cup \cdots \cup V_{k}$ is the index set of all appearing parametric vertices. One advantage of this representation is that it can be computed in polynomial time, even if the dimension $m$ of the parameter space varies:

Lemma 13. The representation (33) can be constructed in polynomial time when the dimension $d$ of the polytopes is fixed (but the dimension $m$ of the parameter space varies).

Proof. This follows from the above discussion; the number of parametric vertices is polynomial when the dimension $d$ of the polytopes is fixed and the dimension $m$ of the parameter space varies.

\subsection{From the generating function to the counting function}

After computing the parametric generating function $g_{P_{\mathbf{q}}}(\mathbf{z})$ of $P_{\mathbf{q}}$, an explicit representation of the parametric counting function $c(\mathbf{q})=\#\left(P_{\mathbf{q}} \cap \mathbf{Z}^{d}\right)$ can be obtained by evaluating the generating function at $\mathbf{1}$, i.e., $c(\mathbf{q})=g_{P_{\mathbf{q}}}(\mathbf{1})$. Care needs to be taken in this evaluation since $\mathbf{1}$ is a pole of each term in $g_{P_{\mathbf{q}}}(\mathbf{z})$. One typically evaluates these rational functions on a curve $t \mapsto \mathbf{z}(t)$ with $\mathbf{z}(0)=\mathbf{1}$ that meets singularities only in finitely many points and then computes the constant terms of the Laurent expansions of the resulting univariate meromorphic functions about $t=0$; see [3, 5, 11, 24].

Applying this process to (32) and (33), one obtains the counting formulas

$$
c(\mathbf{q})=\sum_{i=1}^{k}\left[\tilde{Q}_{i}\right](\mathbf{q}) \sum_{\mathbf{v}_{j} \in V_{i}} c_{\mathbf{v}_{j}(\mathbf{q})+C_{j}}
$$

and

$$
c(\mathbf{q})=\sum_{\mathbf{v}_{j}}\left[\tilde{A}_{j}\right](\mathbf{q}) c_{\mathbf{v}_{j}(\mathbf{q})+C_{j}},
$$

where $c_{\mathbf{v}_{j}(\mathbf{q})+C_{j}}$ is the sum of the constant terms in the Laurent expansions of the terms in $g_{\mathbf{v}_{j}(\mathbf{q})+C_{j}}(\mathbf{z})$.

\subsection{The resulting algorithms}

The complete resulting algorithm, based on a chamber decomposition, is shown below.

\section{Algorithm 14 (Primal parametric Barvinok algorithm).}

Input: full-dimensional parametric polytope $P_{\mathbf{q}}=\left\{\mathbf{x} \in \mathbf{R}^{d}: A \mathbf{x} \leq \mathbf{q}\right\}$, with $\mathbf{q} \in Q \subseteq$ $\mathbf{R}^{m}$; the maximum enumerated cone index $\ell$

Output: parametric counting function $c: Q \rightarrow \mathbf{N}$ with $c(\mathbf{q})=\#\left(P_{\mathbf{q}} \cap \mathbf{Z}^{d}\right)$ 
1. Compute the chamber decomposition $\mathcal{Q} \subset 2^{Q}$ of $P_{\mathbf{q}}$ and for each $Q_{i} \in \mathcal{Q}$ of maximal dimension, the corresponding active vertices $V_{i}=\left\{\mathbf{v}_{j}(\mathbf{q})\right\}_{j}$ (see Section 1.2)

2. For each vertex cone $\mathbf{v}_{j}(\mathbf{q})+C_{j}$ of $P_{\mathbf{q}}$, with $\mathbf{v}_{j}(\mathbf{q}) \in \bigcup_{Q_{i} \in \mathcal{Q}} V_{i}$

(a) Triangulate $C_{j}$ into partially open full-dimensional simplicial cones $\left[C_{j}\right]=$ $\sum_{k}\left[\tilde{C}_{j k}\right]$ (see Section 2.2)

(b) For each $\tilde{C}_{j k}$, apply Barvinok's signed decomposition into partially open fulldimensional cones $\left[\tilde{C}_{j k}\right]=\sum_{l} \varepsilon_{j k l}\left[\tilde{C}_{j k l}\right]$ of index at most $\ell$ (see Section 2.3)

(c) For each $\tilde{C}_{j k l}$, write down the generating function $g_{\mathbf{v}_{j}(\mathbf{q})+\tilde{C}_{j k l}}(\mathbf{z})(27)$ of the affine cone $\mathbf{v}_{j}(\mathbf{q})+\tilde{C}_{j k l}$ (see Section 3.1)

(d) Write down $g_{\mathbf{v}_{j}(\mathbf{q})+C_{j}}(\mathbf{z})=\sum_{k} \sum_{l} \varepsilon_{j k l} g_{\mathbf{v}_{j}(\mathbf{q})+\tilde{C}_{j k l}}(\mathbf{z})$

3. Compute partially open chambers $\tilde{Q}_{i}$ from $Q_{i}$ and write down the generating function $g_{P_{\mathbf{q}}}(\mathbf{z})$ (32) of the parametric polytope $P_{\mathbf{q}}$ (see Section 3.2)

4. Specialize the generating function $g_{P_{\mathbf{q}}}(\mathbf{z})$ to obtain the counting function $c(\mathbf{q})=$ $g_{P_{\mathbf{q}}}(\mathbf{1})$ (see Section 3.3)

We omit the variation based on activity regions, as it is nearly identical.

\section{References}

[1] Velleda Baldoni, Nicole Berline, and Michèle Vergne. Sum of values of a monomial over lattice points of a convex polygon. User's guide, October 2007.

Available from URL http://www. math.polytechnique.fr/ berline/.

[2] M. Welleda Baldoni-Silva, Jesús A. De Loera, and Michèle Vergne. Counting integer flows in networks. Found. Comput. Math., 4(3):277-314, 2004.

[3] Alexander I. Barvinok. A polynomial time algorithm for counting integral points in polyhedra when the dimension is fixed. Math. Oper. Res., 19:769-779, 1994.

[4] Alexander I. Barvinok. Computing the volume, counting integral points, and exponential sums. Discrete Comput. Geom., 10(2):123-141, 1993.

[5] Alexander I. Barvinok and James E. Pommersheim. An algorithmic theory of lattice points in polyhedra. In Louis J. Billera, Anders Björner, Curtis Greene, Rodica E. Simion, and Richard P. Stanley, editors, New Perspectives in Algebraic Combinatorics, volume 38 of Math. Sci. Res. Inst. Publ., pages 91-147. Cambridge Univ. Press, Cambridge, 1999.

[6] Matthias Beck and Frank Sottile. Irrational proofs for three theorems of Stanley. Eur. J. Combin., 28(1):403-409, 2007.

[7] Nicole Berline and Michèle Vergne. Local Euler-Maclaurin formula for polytopes. eprint arXiv:math.CO/0507256, July 2006. 
[8] Michel Brion. Points entiers dans les polyédres convexes. Ann. Sci. École Norm. Sup., 21(4):653-663, 1988.

[9] Michel Brion and Michèle Vergne. Residue formulae, vector partition functions and lattice points in rational polytopes. J. Amer. Math. Soc., 10:797-833, 1997.

[10] Jesús A. De Loera, David Haws, Raymond Hemmecke, Peter Huggins, Bernd Sturmfels, and Ruriko Yoshida. Short rational functions for toric algebra and applications. J. Symb. Comput., 38(2):959-973, 2004.

[11] Jesús A. De Loera, Raymond Hemmecke, Jeremiah Tauzer, and Ruriko Yoshida. Effective lattice point counting in rational convex polytopes. J. Symb. Comput., 38 (4):1273-1302, 2004.

[12] Jesús A. De Loera, David Haws, Raymond Hemmecke, Peter Huggins, Jeremiah Tauzer, and Ruriko Yoshida. LattE, version 1.2, 2005.

Available from URL http://www. math.ucdavis.edu/ latte/.

[13] Elke Eisenschmidt and Matthias Köppe. Integrally indecomposable polytopes and the survivable network design problem. In Proceedings of the 6th International Workshop on the Design of Reliable Communication Networks, DRCN 2007, 2007. To appear.

[14] Israel M. Gelfand, Mikhail M. Kapranov, and Andrei V. Zelevinsky. Discriminants, Resultants and Multidimensional Determinants. Birkhäuser, Boston, 1994.

[15] Matthias Köppe. A primal Barvinok algorithm based on irrational decompositions. SIAM J. Discrete Math., 21(1):220-236, 2007.

[16] Matthias Köppe. LattE macchiato, version 1.2-mk-0.7.1, an improved version of De Loera et al.'s LattE program for counting integer points in polyhedra with variants of Barvinok's algorithm, 2006.

Available from URL http://www.math.uni-magdeburg.de/ mkoeppe/latte/.

[17] Vincent Loechner and Doran K. Wilde. Parameterized polyhedra and their vertices. Int. J. Parallel Prog., 25(6):525-549, December 1997.

[18] Benoît Meister. Stating and Manipulating Periodicity in the Polytope Model. Applications to Program Analysis and Optimization. PhD thesis, ICPS, Université Louis Pasteur de Strasbourg, France, December 2004.

[19] Julian Pfeifle and Jörg Rambau. Computing triangulations using oriented matroids. In Michael Joswig and Nobuki Takayama, editors, Algebra, Geometry, and Software Systems, pages 49-75. Springer, 2003.

[20] Richard P. Stanley. Enumerative Combinatorics, volume I. Cambridge, 1997.

[21] Sven Verdoolaege. barvinok: User guide, 2007. Available from URL http://freshmeat.net/projects/barvinok/.

[22] Sven Verdoolaege and Kevin M. Woods. Counting with rational generating functions. eprint arXiv:math.CO/0504059, May 2006. To appear in J. Symb. Comput.

[23] Sven Verdoolaege, Kevin M. Woods, Maurice Bruynooghe, and Ronald Cools. Computation and manipulation of enumerators of integer projections of parametric poly- 
topes. Report CW 392, Katholieke Universiteit Leuven, Department of Computer Science, March 2005.

[24] Sven Verdoolaege, Rachid Seghir, Kristof Beyls, Vincent Loechner, and Maurice Bruynooghe. Counting integer points in parametric polytopes using Barvinok's rational functions. Algorithmica, 48(1):37-66, June 2007. 